\title{
POSTPROCESSING OF A FINITE VOLUME ELEMENT METHOD FOR SEMILINEAR PARABOLIC PROBLEMS*
}

\author{
Min YAng ${ }^{1}$, Chunjia Bi ${ }^{1}$ And Jiangguo Liu ${ }^{2}$
}

\begin{abstract}
In this paper, we study a postprocessing procedure for improving accuracy of the finite volume element approximations of semilinear parabolic problems. The procedure amounts to solve a source problem on a coarser grid and then solve a linear elliptic problem on a finer grid after the time evolution is finished. We derive error estimates in the $L^{2}$ and $H^{1}$ norms for the standard finite volume element scheme and an improved error estimate in the $H^{1}$ norm. Numerical results demonstrate the accuracy and efficiency of the procedure.
\end{abstract}

Mathematics Subject Classification. 65N30, 65N15.

Received April 25, 2008. Revised December 8, 2008.

Published online June 12, 2009.

\section{INTRODUCTION}

We consider the following semilinear parabolic problem:

$$
\begin{aligned}
u_{t}-\nabla \cdot(A \nabla u) & =f(\mathbf{x}, t, u), \quad \text { in } \Omega \times(0, T], \\
u & =0, \quad \text { on } \partial \Omega \times(0, T], \\
u & =u_{0}, \quad \text { in } \Omega \times\{0\},
\end{aligned}
$$

where $\Omega$ is a bounded polygonal domain in $\mathbb{R}^{2}, u_{0}(\mathbf{x})$ a given smooth function and $A(\mathbf{x})=\left(a_{i j}(\mathbf{x})\right)_{i, j=1}^{2}$, $\left(a_{i j}(\mathbf{x}) \in W^{1, \infty}(\Omega)\right)$ a symmetric and positive definite matrix in $\Omega$, i.e., there exists a positive constant $a_{*}$ such that

$$
0<a_{*}|\zeta|^{2} \leq \zeta^{T} A(\mathbf{x}) \zeta, \quad \forall \zeta \in \mathbb{R}^{2}, \mathbf{x} \in \Omega
$$

We assume that $f(\mathbf{x}, t, u)$ is a real-valued function defined on $\Omega \times(0, T] \times \mathbb{R}$ satisfying the following condition:

$$
|f(\mathbf{x}, t, w)-f(\mathbf{x}, t, v)| \leq C_{f}|w-v|(1+|w|+|v|)^{\gamma}, \quad \forall w, v \in \mathbb{R}, \text { a.e. }(\mathbf{x}, t) \in \Omega \times(0, T] .
$$

Here $C_{f}$ is a positive constant and $0 \leq \gamma<\infty$. For example, $f(\mathbf{x}, t, u)$ could be an arbitrary polynomial of $u$. The condition (1.2) implies that $f$ is locally Lipschitz continuous [21,30]. The problem (1.1) arises

Keywords and phrases. Error estimates, finite volume elements, postprocessing, semilinear parabolic problems.

* M. Yang was supported by the visitor scholarship programme for young faculties of Shandong province.

${ }^{1}$ Department of Mathematics, Yantai University, Yantai, Shandong 264005, P. R. China. yang@ytu.edu.cn; bicj@ytu.edu.cn

2 Department of Mathematics, Colorado State University, Fort Collins, CO 80523-1874, USA. liu@math.colostate.edu 
in many applications, e.g., combustion modeling, epidemic phenomena, and stochastic controls. As in [19,21,31], we suppose that the initial data $u_{0}$ is sufficiently smooth and compatible and the problem (1.1) admits a unique solution satisfying

$$
\max _{0 \leq t \leq T}\|u(\cdot, t)\|_{3, q}+\left\|u_{t}(\cdot, t)\right\|_{3, r} \leq M
$$

where $M$ is a positive constant and $q, r>1$ are constants to be specified in Section 3. A detailed discussion on the regularity of solutions of nonlinear evolution problems can be found in $[20,28,29]$.

Finite volume element (FVE) methods are discretization tools widely used in engineering applications. The methods possess the advantages of local modeling and simple structures and offer the flexibility to handle complicated geometries. More importantly, the methods ensure local mass conservation, a highly desirable property in many applications. We refer to the monographs [16,22] for general presentations of these methods, and to the papers $[2,4,5,11,17,27,32,33]$ (also the references therein) for more details.

To the best of our knowledge, little progress has been made on the FVE solution of problems of the form (1.1). A reason for this might be that the analysis for the nonlinear term is often very involved. For the linear case, a unified approach is presented in [10] to derive error estimates in the $L^{2}, H^{1}$, and $L^{\infty}$ norms by connecting FVE methods with finite element (FE) methods. Error estimates and superconvergence results in the $L^{p}$ norm $(2 \leq p<\infty)$ are obtained in [11]. In [8], FVE methods for two-dimensional linear parabolic problems in convex polygonal domains are studied and error estimates in the $H^{1}, L^{2}$, and $L^{\infty}$ norms under limited regularities of exact solutions are established. In order to solve the discrete equations more efficiently, several symmetric FVE schemes are developed in $[23,25]$.

On the other hand, developing efficient algorithms for finite volume element methods is an interesting problem and has been attracting many researchers' attention. The convergence of a V-cycle multigrid algorithm for a FVE method for variable coefficient elliptic problems is considered in [9]. Two-grid FVE methods are presented in [2] for linear and nonlinear elliptic problems and error estimates are derived to justify efficiency of the algorithms. Residual type a posteriori error estimates and an adaptive strategy for the finite volume approximation are developed in [6] to treat two- and three- dimensional steady-state convection diffusion reaction problems. In [34], a two-level additive Schwarz domain decomposition FVE method is studied and its convergence rates are shown to be optimal and independent of the number of subregions.

The purpose of this paper is to formulate and analyze a postprocessing FVE procedure for the semilinear parabolic initial boundary value problem (1.1). We first prove the optimal order error estimates in the $H^{1}$ and $L^{2}$ norm for the standard FVE scheme under certain regularity assumptions on the solution. The main difficulty for this part is to treat the locally Lipschitz continuous nonlinearity and prove the existence of the numerical solution. Furthermore, we develop a postprocessing algorithm to improve efficiency of the methods. The postprocessing technique can be seen as a novel two-level or two-grid method, which involves an additional solution on a finer grid after the time evolution is finished. Unlike the traditional two-grid or two-level approaches, there is no communication from fine to coarse meshes until the end of time-marching $[13,18,24]$. This means that the extra cost of the postprocessing is relatively negligible when compared with the cost of computations from $t=0$ to $t=T$ on the coarser mesh. In [19], the postprocessed FE methods are proved to have a higher rate of convergence in $H^{1}$ and $L^{2}$ norms than the standard ones when other than piecewise linear elements are used. A postprocessing linear FE scheme is studied in [14] and the improved $H^{1}$ convergence rate is observed. The above analysis is extended to fully discrete case and both temporal and spatial estimates are obtained in [31]. We want to point out that although postprocessing techniques have been studied extensively in the FE framework, how to apply them to FVE methods is still not very well known. There are certain difficulties in handling piecewise constant test functions and nonsymmetric bilinear forms.

The rest of this paper is organized as follows. In Section 2, we describe the FVE method for the semilinear parabolic initial boundary value problem (1.1). In Section 3 we derive optimal order semidiscrete error estimates for finite volume approximation in the $H^{1}$ and $L^{2}$ norms under certain regularity assumptions. The postprocessing FVE procedure and the improved error estimate in $H^{1}$ norm are established in Section 4 . Finally numerical experiments are presented in Section 5 to illustrate the theoretical analysis. 

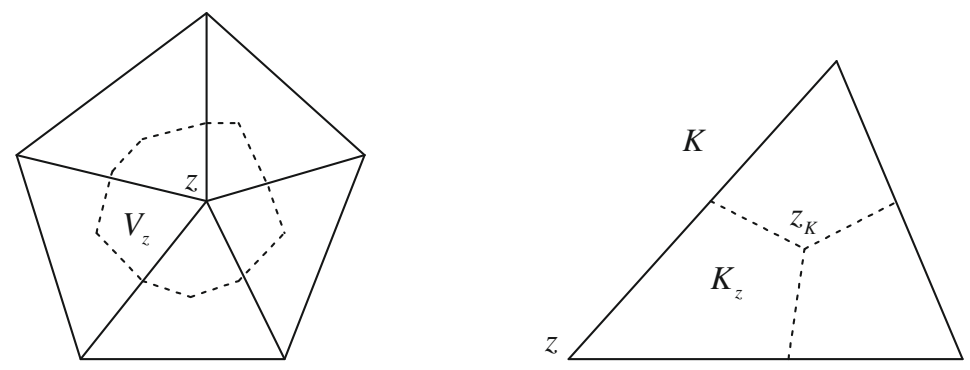

FiguRE 1. Left: A sample region with dotted lines indicating the corresponding control volume $V_{z}$. Right: A triangle $K$ partitioned into three quadrilaterals $K_{z}$.

Throughout this paper we use $C$ and $\epsilon$ to denote a generic positive constant and a generic small positive constant independent of discretization parameters.

\section{Finite volume element scheme}

\subsection{Notations}

We shall use the standard notations for the Sobolev space $W_{p}^{m}(\Omega)$ with the norm $\|\cdot\|_{m, p, \Omega}$ and the seminorm $|\cdot|_{m, p, \Omega}$, see [1]. To simplify the notations, we denote $W_{2}^{m}(\Omega)$ by $H^{m}(\Omega)$ and skip the index $p=2$ and $\Omega$, when there is no ambiguity. That is, $\|u\|_{m, p}=\|u\|_{m, p, \Omega},\|u\|_{m}=\|u\|_{m, 2, \Omega}$. The same convention is adopted for the seminorms as well. We denote by $H_{0}^{1}(\Omega)$ the subspace of $H^{1}(\Omega)$ of functions vanishing on the boundary $\partial \Omega$.

Let $\mathcal{T}_{h}$ be a quasi-uniform triangulation of $\Omega$ with $h=\max h_{K}$, where $h_{K}$ is the diameter of any triangle $K \in \mathcal{T}_{h}$. For this primal triangulation, let $S_{h}$ be the standard conforming finite element space of piecewise linear functions,

$$
S_{h}=\left\{v \in C(\Omega):\left.v\right|_{K} \text { linear, } \forall K \in \mathcal{T}_{h} ;\left.v\right|_{\partial \Omega}=0\right\} .
$$

In order to describe the FVE method, we introduce a dual partition $\mathcal{T}_{h}^{*}$ whose elements are called control volumes. We construct the control volumes in the same way as in $[7,15]$. Let $z_{K}$ be the barycenter of any $K \in \mathcal{T}_{h}$. We connect $z_{K}$ using line segments to the edge midpoints of $K$, and divide $K$ into three quadrilaterals $K_{z}$, $z \in Z_{h}(K)$, where $Z_{h}(K)$ is a set of the vertices of $K$, see Figure 1 . For each vertex $z \in Z_{h}=\cup_{K \in \mathcal{T}_{h}} Z_{h}(K)$, we associate a control volume $V_{z}$, which consists of the union of the subregions $K_{z}$ sharing the vertex $z$. Thus we obtain a group of control volumes covering the domain $\Omega$. This is the dual partition $\mathcal{T}_{h}^{*}$. We denote the set of interior vertices of $Z_{h}$ by $Z_{h}^{0}$.

A dual partition $\mathcal{T}_{h}^{*}$ is regular or quasi-uniform, if there exists a positive constant $C>0$ such that

$$
C^{-1} h^{2} \leq \operatorname{meas}\left(V_{z}\right) \leq C h^{2}, \forall V_{z} \in \mathcal{T}_{h}^{*}
$$

We want to point out that a barycenter-type dual partition can be constructed for any finite element triangulation $\mathcal{T}_{h}$ and involves relatively simple calculations. In addition, if the primal triangulation $\mathcal{T}_{h}$ is quasi-uniform, then the dual partition $\mathcal{T}_{h}^{*}$ is also quasi-uniform. 


\subsection{Construction of FVE scheme}

We formulate the FVE method for the problem (1.1). Given a vertex $z \in Z_{h}^{0}$, we integrate (1.1) over the associated control volume $V_{z}$ and apply the Green's formula to obtain

$$
\int_{V_{z}} u_{t} \mathrm{~d} x-\int_{\partial V_{z}}(A \nabla u) \cdot \mathbf{n} \mathrm{d} s=\int_{V_{z}} f(\mathbf{x}, t, u) \mathrm{d} x,
$$

where $n$ denotes the unit outer normal vector to $\partial V_{z}$. It should be noted that the above formulation is a way of stating that we have an integral conservation form on the control volume.

The integral relation (2.1) can be written in a variational form similar to that of the finite element method with the help of an interpolation operator $I_{h}^{*}: S_{h} \rightarrow S_{h}^{*}$ defined by

$$
I_{h}^{*} v=\sum_{z \in Z_{h}^{0}} v(z) \Psi_{z}
$$

where

$$
S_{h}^{*}=\left\{v \in L^{2}(\Omega):\left.v\right|_{V_{z}} \text { constant, } \forall z \in Z_{h}^{0} ;\left.v\right|_{V_{z}}=0, \forall z \in \partial \Omega\right\},
$$

and $\Psi_{z}$ is the characteristic function of the control volume $V_{z}$. It was shown in [11] that

$$
\left\|v_{h}-I_{h}^{*} v_{h}\right\|_{0, p} \leq C h^{s}\left\|v_{h}\right\|_{s, p}, \quad s=0,1
$$

and in [7] that

$$
\left\|I_{h}^{*} v_{h}\right\|_{0, p} \leq C\left\|v_{h}\right\|_{0, p}
$$

for all $v_{h} \in S_{h}$ and $p>1$. Furthermore, $\left(v_{h}, I_{h}^{*} w_{h}\right)$ is symmetric and positive definite for any $v_{h}, w_{h} \in S_{h}$. Therefore, it defines an inner product on $S_{h}$, and the corresponding discrete norm is equivalent to the $L^{2}$ norm. In other words, there exist two constants $C_{*}>0$ and $C^{*}>0$ independent of $h$ such that

$$
C_{*}\left\|v_{h}\right\|_{0} \leq\left\|\mid v_{h}\right\|\left\|_{0} \leq C^{*}\right\| v_{h} \|_{0}, \quad \forall v_{h} \in S_{h},
$$

with $\left\|\left|v_{h} \|\right|_{0}=\left(v_{h}, I_{h}^{*} v_{h}\right)^{1 / 2}\right.$.

For any $I_{h}^{*} v_{h}$, we multiply $(2.1)$ by $v_{h}(z)$ and sum over all $z \in Z_{h}^{0}$ to obtain

$$
\left(u_{t}, I_{h}^{*} v_{h}\right)+a_{h}\left(u, I_{h}^{*} v_{h}\right)=\left(f(\mathbf{x}, t, u), I_{h}^{*} v_{h}\right), \quad \forall v_{h} \in S_{h},
$$

where the bilinear form $a_{h}\left(\cdot, I_{h}^{*} \cdot\right)$ is defined as: for any $u \in H_{0}^{1}(\Omega), v_{h} \in S_{h}$,

$$
a_{h}\left(u, I_{h}^{*} v_{h}\right)=-\sum_{z \in Z_{h}^{0}} v_{h}(z) \int_{\partial V_{z}}(A \nabla u) \cdot \mathbf{n d} s .
$$

Our semidiscrete FVE method for problem (1.1) is to find $u_{h}(t) \in S_{h}$ for all $0 \leq t \leq T$ such that

$$
\left(u_{h, t}, I_{h}^{*} v_{h}\right)+a_{h}\left(u_{h}, I_{h}^{*} v_{h}\right)=\left(f\left(\mathbf{x}, t, u_{h}\right), I_{h}^{*} v_{h}\right), \quad \forall v_{h} \in S_{h}
$$

with the initial approximation given by

$$
u_{h}(0)=R_{h} u_{0}
$$

where $R_{h}: H_{0}^{1}(\Omega) \rightarrow S_{h}$ denotes the elliptic projector satisfying

$$
a_{h}\left(R_{h} u, I_{h}^{*} v_{h}\right)=a_{h}\left(u, I_{h}^{*} v_{h}\right), \quad \forall v_{h} \in S_{h} .
$$


In $[10,11,15]$, it was proved that

$$
\begin{gathered}
\left\|u-R_{h} u\right\|_{1} \leq C h\|u\|_{2}, \quad \forall u \in H^{2}(\Omega) \cap H_{0}^{1}(\Omega), \\
\left\|u-R_{h} u\right\|_{0, p} \leq C h^{2}\|u\|_{3, q}, \quad \forall u \in W_{q}^{3}(\Omega) \cap H_{0}^{1}(\Omega),
\end{gathered}
$$

where $q>1$ if $p=2$, and $q=2 p /(p+2)$ if $p>2$.

Remark 2.1. Let $\left\{\Phi_{z}: z \in Z_{h}^{0}\right\}$ be the standard basis functions of $S_{h},\left\{\Psi_{z}: z \in Z_{h}^{0}\right\}$ be the associated basis of $S_{h}^{*}$, and $u_{h}(t)=\sum_{z \in Z_{h}^{0}} \alpha_{z}(t) \Phi_{z}$. Then scheme (2.6) can be written as a system of ordinary differential equations

$$
M \alpha^{\prime}(t)+S \alpha(t)=\tilde{f}(t, \alpha(t)), 0 \leq t \leq T ; \alpha(0)=\beta,
$$

where $M=\left(\left(\Phi_{z}, \Psi_{w}\right)\right)_{z w}$ and $S=\left(a_{h}\left(\Phi_{z}, \Psi_{w}\right)\right)_{z w}$ are the mass and stiffness matrices, respectively, and $\alpha(t)$ and $\beta$ vectors of the nodal values of $u_{h}(t)$ and $R_{h} u_{0}$. Thus scheme (2.6) represents a non-autonomous system of ordinary differential equations with a locally Lipschitz continuous right-hand side. From (2.4) and Lemma 3.1 below, we know that $M$ is symmetric, and both $M$ and $S$ are positive definite. This implies that there exists a unique local solution $u_{h}$ on a certain maximal subinterval $\left[0, t_{* *}\right)$ of $[0, T]$. We will show in Lemma 3.4 that $t_{* *}=T$ for sufficiently small $h$.

\section{ERror ANALYSis of THE FINITE VOLUME ELEMENT SCHEME}

We will frequently use the following Sobolev's inequality [1]: for $p \in[1, \infty)$, there exists a constant $C=$ $C(\Omega, p)$ such that

$$
\|v\|_{0, q} \leq C\|v\|_{s, p}, \quad \frac{1}{p} \geq \frac{1}{q} \geq \frac{1}{p}-\frac{s}{2}, \forall v \in W_{p}^{s}(\Omega)
$$

Since $\mathcal{T}_{h}$ is quasi-uniform, the following inverse estimate holds for all $v \in S_{h}$, see [3,12]:

$$
\|v\|_{m, p} \leq C h^{l-m-2\left(\frac{1}{q}-\frac{1}{p}\right)}\|v\|_{l, q}, \quad 0 \leq l \leq m \leq 1,1 \leq q \leq p \leq \infty .
$$

The following two lemmas have been proved in [10], where Lemma 3.1 indicates that the bilinear form $a_{h}\left(\cdot, I_{h}^{*} \cdot\right)$ is continuous and coercive on $S_{h}$, while Lemma 3.2 shows that $a_{h}\left(\cdot, I_{h}^{*} \cdot\right)$ is generally unsymmetric but not too far away from being symmetric.

Lemma 3.1. For $h$ sufficiently small, there exist two positive constants $C^{*}$ and $C_{*}$ independent of $h$ such that

$$
\begin{aligned}
\left|a_{h}\left(w_{h}, I_{h}^{*} v_{h}\right)\right| & \leq C^{*}\left\|w_{h}\right\|_{1}\left\|v_{h}\right\|_{1}, \quad \forall w_{h}, v_{h} \in S_{h}, \\
a_{h}\left(v_{h}, I_{h}^{*} v_{h}\right) & \geq C_{*}\left\|v_{h}\right\|_{1}^{2}, \quad \forall v_{h} \in S_{h} .
\end{aligned}
$$

Lemma 3.2. For $h$ sufficiently small, there exists a constant $C>0$ such that

$$
\left|a_{h}\left(w_{h}, I_{h}^{*} v_{h}\right)-a_{h}\left(v_{h}, I_{h}^{*} w_{h}\right)\right| \leq C h\left\|w_{h}\right\|_{1}\left\|v_{h}\right\|_{1}, \quad \forall w_{h}, v_{h} \in S_{h} .
$$

In [21], the following result has been established regarding the local Lipschitz continuity of $f$ as a mapping from $L^{2(\gamma+1)}(\Omega)$ to $L^{2}(\Omega)$.

Lemma 3.3. Suppose that $f$ satisfies the condition (1.2). Then there exists a positive constant $C=C\left(\gamma, C_{f}, \Omega\right)$ such that

$$
\|f(\mathbf{x}, t, w)-f(\mathbf{x}, t, v)\|_{0} \leq C\|w-v\|_{0,2(\gamma+1)}\left(1+\|w\|_{0,2(\gamma+1)}^{\gamma}+\|v\|_{0,2(\gamma+1)}^{\gamma}\right)
$$

for all $w, v \in L^{2(\gamma+1)}(\Omega)$ and a.e. $(\mathbf{x}, t) \in \Omega \times(0, T]$. 
Now we state the first main result that estimates the $H^{1}$ norm error between the elliptic projection of the exact solution and the FVE approximation. It also asserts the existence of an approximation solution $u_{h}(t)$ in the whole time period $[0, T]$.

Lemma 3.4. Let $u$ and $u_{h}$ be the solutions of (1.1) and (2.6), respectively. Let $R_{h} u$ be the elliptic projection of $u$ onto $S_{h}$ defined in (2.7). Suppose that (1.2) and (1.3) hold. Then, there exists $h_{0}>0$ and a positive constant $C=C\left(\gamma, C_{f}, \Omega, M\right)$ independent of the discretization parameter, such that for all $h \in\left(0, h_{0}\right]$ and $t \in[0, T]$,

$$
\left\|R_{h} u-u_{h}\right\|_{1} \leq C h^{2} .
$$

Proof. We decompose the error as $u_{h}-u=\xi-\eta$, where $\xi=u_{h}-R_{h} u$ and $\eta=u-R_{h} u$. According to Remark 2.1, there exists a maximal interval $\left[0, t_{* *}\right) \subset[0, T]$ such that $u_{h}(t)$ exists for all $t \in\left[0, t_{* *}\right)$. Here either $t_{* *}=T$ or $t_{* *}<T$, and $\lim _{t \rightarrow t_{* *}}\left|u_{h}(t)\right|=+\infty$. We will show below that $t_{* *}=T$, if $h$ is small enough.

From (2.5) and (2.6), we have the following error equation

$$
\left(\xi_{t}, I_{h}^{*} v_{h}\right)+a_{h}\left(\xi, I_{h}^{*} v_{h}\right)=\left(\eta_{t}, I_{h}^{*} v_{h}\right)+\left(f\left(\mathbf{x}, t, u_{h}\right)-f(\mathbf{x}, t, u), I_{h}^{*} v_{h}\right), \quad t \in\left(0, t_{* *}\right) .
$$

Taking $v_{h}=\xi_{t}$ in (3.8) leads to

$$
\left\|\left|\xi_{t} \|\right|_{0}^{2}+\frac{1}{2} \frac{\mathrm{d}}{\mathrm{d} t} a_{h}\left(\xi, I_{h}^{*} \xi\right)=\frac{1}{2}\left[a_{h}\left(\xi_{t}, I_{h}^{*} \xi\right)-a_{h}\left(\xi, I_{h}^{*} \xi_{t}\right)\right]+\left(\eta_{t}, I_{h}^{*} \xi_{t}\right)+\left(f\left(\mathbf{x}, t, u_{h}\right)-f(\mathbf{x}, t, u), I_{h}^{*} \xi_{t}\right) .\right.
$$

It follows from Lemma 3.2 and the inverse estimate (3.2) that

$$
\left|a_{h}\left(\xi_{t}, I_{h}^{*} \xi\right)-a_{h}\left(\xi, I_{h}^{*} \xi_{t}\right)\right| \leq C h\|\xi\|_{1}\left\|\xi_{t}\right\|_{1} \leq C\|\xi\|_{1}\left\|\xi_{t}\right\|_{0} \leq C\|\xi\|_{1}^{2}+\epsilon\left\|\xi_{t}\right\|_{0}^{2} .
$$

By $(2.3)$,

$$
\begin{aligned}
\left|\left(\eta_{t}, I_{h}^{*} \xi_{t}\right)\right|+\left|\left(f\left(\mathbf{x}, t, u_{h}\right)-f(\mathbf{x}, t, u), I_{h}^{*} \xi_{t}\right)\right| & \leq\left\|\eta_{t}\right\|_{0}\left\|I_{h}^{*} \xi_{t}\right\|_{0}+\left\|f\left(\mathbf{x}, t, u_{h}\right)-f(\mathbf{x}, t, u)\right\|_{0}\left\|I_{h}^{*} \xi_{t}\right\|_{0} \\
& \leq C\left(\left\|\eta_{t}\right\|_{0}^{2}+\left\|f\left(\mathbf{x}, t, u_{h}\right)-f(\mathbf{x}, t, u)\right\|_{0}^{2}\right)+\epsilon\left\|\xi_{t}\right\|_{0}^{2} .
\end{aligned}
$$

Thus, using (2.4) and choosing $\epsilon$ small enough, we obtain

$$
\begin{aligned}
\frac{\mathrm{d}}{\mathrm{d} t} a_{h}\left(\xi, I_{h}^{*} \xi\right) & \leq C\left(\left\|\eta_{t}\right\|_{0}^{2}+\left\|f\left(\mathbf{x}, t, u_{h}\right)-f(\mathbf{x}, t, u)\right\|_{0}^{2}+\|\xi\|_{1}^{2}\right) \\
& \leq C\left(\left\|\eta_{t}\right\|_{0}^{2}+\left\|f\left(\mathbf{x}, t, u_{h}\right)-f\left(\mathbf{x}, t, R_{h} u\right)\right\|_{0}^{2}+\left\|f\left(\mathbf{x}, t, R_{h} u\right)-f(\mathbf{x}, t, u)\right\|_{0}^{2}+\|\xi\|_{1}^{2}\right),
\end{aligned}
$$

for all $t \in\left(0, t_{* *}\right)$. By Lemma 3.3, the Sobolev's inequality (3.1) and (2.8), we have

$$
\begin{aligned}
\left\|f\left(\mathbf{x}, t, R_{h} u\right)-f(\mathbf{x}, t, u)\right\|_{0} & \leq C\|\eta\|_{0,2(\gamma+1)}\left(1+\|u\|_{0,2(\gamma+1)}^{\gamma}+\left\|R_{h} u\right\|_{0,2(\gamma+1)}^{\gamma}\right) \\
& \leq C\|\eta\|_{0,2(\gamma+1)}\left(1+\|u\|_{1}^{\gamma}+\left\|R_{h} u\right\|_{1}^{\gamma}\right) \\
& \leq C\|\eta\|_{0,2(\gamma+1)}\left(1+\|u\|_{1}^{\gamma}+\left(\|u\|_{1}+h\|u\|_{2}\right)^{\gamma}\right) \\
& \leq C\|\eta\|_{0,2(\gamma+1)} .
\end{aligned}
$$

On the other hand, by a similar argument as above, we have

$$
\left\|f\left(\mathbf{x}, t, R_{h} u\right)-f\left(\mathbf{x}, t, u_{h}\right)\right\|_{0} \leq C\|\xi\|_{1}\left(1+\|\xi\|_{1}^{\gamma}\right) .
$$

Note that $\xi(0)=0$. By the continuity of $\xi(t)$, we set $t_{*} \in\left(0, t_{* *}\right]$ to be the largest time such that $u_{h}$ exists and $\|\xi(t)\|_{1} \leq 1$ for all $t \in\left[0, t_{*}\right]$. Next we shall show that $t_{*}=T$, if $h$ is small enough. That also means $t_{* *}=T$. 
Now for all $t \in\left(0, t_{*}\right]$, we have

$$
\left\|f\left(\mathbf{x}, t, R_{h} u\right)-f\left(\mathbf{x}, t, u_{h}\right)\right\|_{0} \leq C\|\xi\|_{1}
$$

where $C>0$ is a constant depending on the norms of $u$ over the time interval $\left[0, t^{*}\right]$.

By (3.9)-(3.11), we get

$$
\frac{\mathrm{d}}{\mathrm{d} t} a_{h}\left(\xi, I_{h}^{*} \xi\right) \leq C\left(\left\|\eta_{t}\right\|_{0}^{2}+\|\eta\|_{0,2(\gamma+1)}^{2}+\|\xi\|_{1}^{2}\right), \quad t \in\left(0, t_{*}\right] .
$$

Integrating (3.12) from 0 to $t \leq t_{*}$, noting $\xi(0)=0$, and using Lemma 3.1 (coercivity), we have

$$
\begin{aligned}
C_{*}\|\xi(t)\|_{1}^{2} & \leq a_{h}\left(\xi(t), I_{h}^{*} \xi(t)\right) \\
& \leq C \int_{0}^{t}\left(\left\|\eta_{t}(s)\right\|_{0}^{2}+\|\eta(s)\|_{0,2(\gamma+1)}^{2}+\|\xi(s)\|_{1}^{2}\right) \mathrm{d} s, \quad t \in\left[0, t_{*}\right] .
\end{aligned}
$$

Then the Gronwall's inequality and (2.9) imply that

$$
\begin{aligned}
\|\xi(t)\|_{1}^{2} & \leq C \int_{0}^{t}\left(\|\eta(s)\|_{0,2(\gamma+1)}^{2}+\left\|\eta_{t}(s)\right\|_{0}^{2}\right) \mathrm{d} s \\
& \leq C h^{4} \int_{0}^{t}\left(\|u(s)\|_{3, q}^{2}+\left\|u_{t}(s)\right\|_{3, r}^{2}\right) \mathrm{d} s, \quad t \in\left[0, t_{*}\right]
\end{aligned}
$$

where $q>1$ if $\gamma=0, q=2(\gamma+1) /(\gamma+2)$ if $\gamma>0$, and $r>1$. Hence,

$$
\|\xi(t)\|_{1} \leq C h^{2}, \quad t \in\left[0, t_{*}\right] .
$$

Therefore, there exists a sufficiently small $h_{0}$ such that for $h \in\left(0, h_{0}\right],\|\xi(t)\|_{1} \leq C h^{2}<1, t \in\left[0, t_{*}\right]$. It follows from the continuity of the function $\|\xi(t)\|_{1}$ that $t_{*}=t_{* *}$. Otherwise, by continuity there must be a $t_{*}^{\prime}$, $t_{*}<t_{*}^{\prime} \leq t_{* *}$ such that $\|\xi(t)\|_{1} \leq 1$ for all $t \in\left[0, t_{*}^{\prime}\right]$. But this contradicts the definition of $t_{*}$.

Now for $h \in\left(0, h_{0}\right]$, we have $\|\xi(t)\|_{1} \leq C h^{2}<1, t \in\left[0, t_{* *}\right]$. We shall show that $t_{* *}=T$. Suppose that $t_{* *}<T$. Then by the definition of $t_{* *}$, we have $\lim _{t \rightarrow t_{* *}}\left|u_{h}(t)\right|_{\infty}=+\infty$. But on the contrary, it follows from a triangle inequality and (3.13) that

$$
\begin{aligned}
\lim _{t \rightarrow t_{* *}}\left|u_{h}(t)\right|_{\infty} & \leq \lim _{t \rightarrow t_{* *}}\left(|\xi(t)|_{\infty}+\left|R_{h} u(t)\right|_{\infty}\right) \\
& \leq \lim _{t \rightarrow t_{* *}} C|\ln h|^{1 / 2}\|\xi(t)\|_{1}+\left|R_{h} u\left(t_{* *}\right)\right|_{\infty} \\
& \leq C|\ln h|^{1 / 2} h^{2}+\left|R_{h} u\left(t_{* *}\right)\right|_{\infty} \leq \text { const. }
\end{aligned}
$$

where we have used the asymptotic Sobolev's inequality $\left\|v_{h}\right\|_{0, \infty} \leq C|\ln h|^{1 / 2}\left\|v_{h}\right\|_{1}, v_{h} \in S_{h}$ (see [26]). Thus it must be $t_{* *}=T$, i.e., the FVE approximation $u_{h}$ exists on the whole of $[0, T]$. From the argument above, we have that for $h \in\left(0, h_{0}\right]$,

$$
\left\|R_{h} u(t)-u_{h}(t)\right\|_{1}=\|\xi(t)\|_{1} \leq C h^{2}, \quad t \in[0, T]
$$

which gives the desired result.

Remark 3.5. We can see from the above lemma that the presence of a locally Lipschitz nonlinearity, satisfying a certain growth condition, leads to certain difficulties in the error analysis but will not degrade the convergence rate observed in the linear case. Moreover, unlike the linear case, we have to prove the existence of an approximate solution on the entire time interval. Some techniques such as using (asymptotic) Sobolev's inequalities and the bootstrap argument play a crucial role in the proof. A similar proof was presented in $[17,21]$. 
By (2.8), (2.9), and Lemma 3.4, we can derive the following $H^{1}$ and $L^{2}$ error estimates for the finite volume element scheme.

Theorem 3.6. Let $u$ and $u_{h}$ be the solutions of (1.1) and (2.6), respectively. Assume the condition in Lemma 3.4 holds. Then for all $h \in\left(0, h_{0}\right]$ and $t \in[0, T]$, we have

$$
\left\|u-u_{h}\right\|_{0}+h\left\|u-u_{h}\right\|_{1} \leq C h^{2},
$$

where $C=C\left(\gamma, C_{f}, \Omega, M\right)$ is independent of the discretization parameter.

\section{Postprocessing And its ERror analysis}

In this section, we present the postprocessing finite volume element algorithm for the semilinear parabolic problem (1.1) based on two finite element spaces. There are two quasi-uniform triangulations $\mathcal{T}_{H}$ and $\mathcal{T}_{h}$, with two different mesh sizes $H$ and $h(H>h)$. The corresponding finite element spaces $S_{H}$ and $S_{h}$ satisfy $S_{H} \subset S_{h}$ and are called the coarser and the finer spaces, respectively.

Suppose that we are interested in the solution of (1.1) at time $T$. Then the idea of our postprocessing technique is to solve the semilinear parabolic problem on a coarser grid $T_{H}$ from $(0, T]$ and then solve a symmetric linear elliptic problem on a finer grid $\mathcal{T}_{h}$ only once, at $t=T$.

In order to present the postprocessing FVE scheme, we introduce the following auxiliary bilinear form: for any $u \in H_{0}^{1}(\Omega), v_{h} \in S_{h}$,

$$
\bar{a}_{h}\left(u, I_{h}^{*} v_{h}\right)=-\sum_{z \in Z_{h}^{0}} v_{h}(z) \int_{\partial V_{z}}(\bar{A} \nabla u) \cdot \mathbf{n} \mathrm{d} s
$$

where $\left.\bar{A}\right|_{K}=A_{K}$, and

$$
A_{K}=\frac{1}{\operatorname{meas}(K)} \int_{K} A(\mathbf{x}) \mathrm{d} x, \quad \forall K \in \mathcal{T}_{h} .
$$

The following lemma has been proved in $[10,15]$.

Lemma 4.1. For any $w_{h}, v_{h} \in S_{h}$, we have

$$
\bar{a}_{h}\left(w_{h}, I_{h}^{*} v_{h}\right)=a\left(w_{h}, v_{h}\right)
$$

where $a(\cdot, \cdot)$ is the bilinear form related to the finite element method, i.e.,

$$
a\left(w_{h}, v_{h}\right)=\int_{\Omega} A \nabla w_{h} \cdot \nabla v_{h} \mathrm{~d} x .
$$

Our postprocessing FVE procedure reads as:

(1) Find $u_{H}(t) \in S_{H}$ such that, for any $v_{H} \in S_{H}$,

$$
\left(u_{H, t}, I_{H}^{*} v_{H}\right)+a_{H}\left(u_{H}, I_{H}^{*} v_{H}\right)=\left(f\left(\mathbf{x}, t, u_{H}\right), I_{H}^{*} v_{H}\right), \quad t \in(0, T] .
$$

We take $u_{H}(0)=R_{H} u_{0}$ as an initial approximation. 
(2) Find $u_{h} \in S_{h}$ such that for any $v_{h} \in S_{h}$,

$$
\bar{a}_{h}\left(u_{h}, I_{h}^{*} v_{h}\right)=-\left(u_{H, t}, I_{h}^{*} v_{h}\right)+\left(f\left(\mathbf{x}, t, u_{H}\right), I_{h}^{*} v_{h}\right), \quad t=T .
$$

We know from Lemma 3.2 that the matrix of $a_{h}\left(v_{h}, I_{h}^{*} w_{h}\right)$ is generally nonsymmetric. This introduces some difficulties in real implementations and the method suitable for symmetric linear systems cannot be used in this case. From Lemma 4.1, we know that the coefficient matrix of the linear system in the second step of the postprocessing procedure is symmetric and positive definite and hence easier to solve. For example, the conjugate gradient methods can be applied effectively.

In [2], the following lemma reveals the difference between the bilinear form of FVE method and that of finite element method.

Lemma 4.2. For any $w_{h}, v_{h} \in S_{h}, w \in H^{2}(\Omega), p \geq 1,1 / p+1 / q=1$, we have

$$
\left|a\left(w_{h}, v_{h}\right)-a_{h}\left(w_{h}, I_{h}^{*} v_{h}\right)\right| \leq C h^{2}\left(h^{-1}\left|w-w_{h}\right|_{1, p}+\|w\|_{2, p}\right)\left\|v_{h}\right\|_{1, q} .
$$

Next we next state and prove three more technical lemmas to be used in the error analysis of the postprocessing FVE scheme.

Lemma 4.3. Let $u$ and $u_{h}$ be the solutions of (1.1) and (2.6), respectively. Suppose that (1.2) and (1.3) hold. Then there exists a positive constant $C=C\left(\gamma, C_{f}, \Omega, M\right)$ such that

$$
\left\|u_{t}-u_{h, t}\right\|_{0} \leq C h, \quad t \in[0, T] .
$$

Proof. Taking $v_{h}=\xi_{t}$ in (3.8), and using (2.3) and the inverse estimate (3.2), we obtain

$$
\begin{aligned}
\left.\left\|\xi_{t}\right\|\right|_{0} ^{2} & \leq C\|\xi\|_{1}\left\|\xi_{t}\right\|_{1}+\left\|\eta_{t}\right\|_{0}\left\|I_{h}^{*} \xi_{t}\right\|_{0}+\left\|f(\mathbf{x}, t, u)-f\left(\mathbf{x}, t, u_{h}\right)\right\|_{0}\left\|I_{h}^{*} \xi_{t}\right\|_{0} \\
& \leq C\left\|\xi_{t}\right\|_{0}\left(\|\xi\|_{1} h^{-1}+\left\|\eta_{t}\right\|_{0}+\left\|f(\mathbf{x}, t, u)-f\left(\mathbf{x}, t, u_{h}\right)\right\|_{0}\right) .
\end{aligned}
$$

By Theorem 3.6,

$$
\left\|u_{h}\right\|_{1} \leq\|u\|_{1}+\left\|u-u_{h}\right\|_{1} \leq \text { const. }
$$

Taking into account Lemma 3.3 and the Sobolev's inequality, we have

$$
\begin{aligned}
\left\|f(\mathbf{x}, t, u)-f\left(\mathbf{x}, t, u_{h}\right)\right\|_{0} & \leq C\left\|u-u_{h}\right\|_{0,2(\gamma+1)}\left(1+\|u\|_{0,2(\gamma+1)}^{\gamma}+\left\|u_{h}\right\|_{0,2(\gamma+1)}^{\gamma}\right) \\
& \leq C\left\|u-u_{h}\right\|_{1}\left(1+\|u\|_{1}^{\gamma}+\left\|u_{h}\right\|_{1}^{\gamma}\right) \\
& \leq C\left\|u-u_{h}\right\|_{1} .
\end{aligned}
$$

Combining (4.7) with (4.9), and using (2.4), (2.9), Lemma 3.4, and Theorem 3.6, we obtain

$$
\left\|\xi_{t}\right\|_{0} \leq C\left(\|\xi\|_{1} h^{-1}+\left\|\eta_{t}\right\|_{0}+\left\|u-u_{h}\right\|_{1}\right) \leq C\left(h+h^{2}\right) .
$$

Combined with (2.9), this finishes the proof.

Lemma 4.4. Let $u$ and $u_{h}$ be the solutions of (1.1) and (2.6), respectively. Suppose that (1.2) and (1.3) hold. Then there exists a positive constant $C=C\left(\gamma, C_{f}, \Omega, M\right)$ such that

$$
\left|\left(f(\mathbf{x}, t, u)-f\left(\mathbf{x}, t, u_{h}\right), I_{h}^{*} v_{h}\right)\right| \leq C h^{2}\left\|v_{h}\right\|_{1},
$$

for any $v_{h} \in S_{h}$ and $t \in[0, T]$. 
Proof. Let $1 / p+1 / q=1 / 2$ with $p=2(1+\gamma)$. By (1.2), the Hölder's inequality, and (2.3), we obtain

$$
\begin{aligned}
\left|\left(f(\mathbf{x}, t, u)-f\left(\mathbf{x}, t, u_{h}\right), I_{h}^{*} v_{h}\right)\right| & \leq C_{f} \int_{\Omega}\left|u-u_{h}\right|\left(1+|u|+\left|u_{h}\right|\right)^{\gamma}\left|I_{h}^{*} v_{h}\right| \mathrm{d} x \\
& \leq C_{f}\left\|u-u_{h}\right\|_{0}\left\|\left(1+|u|+\left|u_{h}\right|\right)^{\gamma}\right\|_{0, q}\left\|I_{h}^{*} v_{h}\right\|_{0, p} \\
& \leq C\left\|u-u_{h}\right\|_{0}\left\|\left(1+|u|+\left|u_{h}\right|\right)^{\gamma}\right\|_{0, q}\left\|v_{h}\right\|_{0, p} .
\end{aligned}
$$

Since $1 / q=(p-2) / 2 p=\gamma / p$, we have

$$
\begin{aligned}
\left\|\left(1+|u|+\left|u_{h}\right|\right)^{\gamma}\right\|_{0, q} & =\left\|1+|u|+\left|u_{h}\right|\right\|_{0, p}^{\gamma} \\
& \leq\left(1+\|u\|_{0, p}+\left\|u_{h}\right\|_{0, p}\right)^{\gamma} .
\end{aligned}
$$

Note that $p \geq 2$. Using the above two estimates and the Sobolev's inequality (3.1), we obtain

$$
\begin{aligned}
\left|\left(f(\mathbf{x}, t, u)-f\left(\mathbf{x}, t, u_{h}\right), I_{h}^{*} v_{h}\right)\right| & \leq C\left\|u-u_{h}\right\|_{0}\left(1+\|u\|_{0, p}+\left\|u_{h}\right\|_{0, p}\right)^{\gamma}\left\|v_{h}\right\|_{0, p} \\
& \leq C\left\|u-u_{h}\right\|_{0}\left\|\left(1+\|u\|_{1}+\left\|u_{h}\right\|_{1}\right)^{\gamma}\right\| v_{h} \|_{1} .
\end{aligned}
$$

Combining Theorem 3.6 and (4.8) leads to

$$
\left|\left(f(\mathbf{x}, t, u)-f\left(\mathbf{x}, t, u_{h}\right), I_{h}^{*} v_{h}\right)\right| \leq C\left\|u-u_{h}\right\|_{0}\left\|v_{h}\right\|_{1} \leq C h^{2}\left\|v_{h}\right\|_{1},
$$

which gives the desired result.

Lemma 4.5. Let $u$ and $u_{h}$ be the solutions of (1.1) and (2.6), respectively. Suppose that (1.2) and (1.3) hold. Then there exists a positive constant $C=C\left(\gamma, C_{f}, \Omega, M\right)$ such that

$$
\left|\left(u_{t}-u_{h, t}, I_{h}^{*} v_{h}\right)\right| \leq C h^{2}\left\|v_{h}\right\|_{1},
$$

for any $v_{h} \in S_{h}$ and $t \in(0, T]$.

Proof. From (2.5), (2.6), and Lemma 3.1 (continuity), we have

$$
\begin{aligned}
\left|\left(u_{t}-u_{h, t}, I_{h}^{*} v_{h}\right)\right| & =\left|a_{h}\left(R_{h} u-u_{h}, I_{h}^{*} v_{h}\right)+\left(f\left(\mathbf{x}, t, u_{h}\right)-f(\mathbf{x}, t, u), I_{h}^{*} v_{h}\right)\right| \\
& \leq\left|a_{h}\left(R_{h} u-u_{h}, I_{h}^{*} v_{h}\right)\right|+\left|\left(f\left(\mathbf{x}, t, u_{h}\right)-f(\mathbf{x}, t, u), I_{h}^{*} v_{h}\right)\right| \\
& \leq C^{*}\left\|R_{h} u-u_{h}\right\|_{1}\left\|v_{h}\right\|_{1}+\left|\left(f\left(\mathbf{x}, t, u_{h}\right)-f(\mathbf{x}, t, u), I_{h}^{*} v_{h}\right)\right| .
\end{aligned}
$$

Therefore, Lemmas 3.4 and 4.4 together with (4.12) yield the desired estimate.

Now comes the main result of this section.

Theorem 4.6. Let $u(T)$ be the solution of (1.1) at time $T$ and $u_{h}$ be the solution of (4.3) and (4.4). Assume that (1.2) and (1.3) hold. Then, there exists a positive constant $C=C\left(\gamma, C_{f}, \Omega, M\right)$ independent of the discretization parameters such that

$$
\left\|u(T)-u_{h}\right\|_{1} \leq C\left(h+H^{2}\right) .
$$


Proof. We drop the explicit dependence on $\mathbf{x}$ and $T$ for conciseness. From (2.5), (4.3), and (4.4), we have

$$
\begin{aligned}
\bar{a}_{h}\left(R_{h} u-u_{h}, I_{h}^{*} v_{h}\right) & =\left(\bar{a}_{h}\left(R_{h} u, I_{h}^{*} v_{h}\right)-a_{h}\left(R_{h} u, I_{h}^{*} v_{h}\right)\right)+a_{h}\left(R_{h} u, I_{h}^{*} v_{h}\right)-\bar{a}_{h}\left(u_{h}, I_{h}^{*} v_{h}\right) \\
& =\left(\bar{a}_{h}\left(R_{h} u, I_{h}^{*} v_{h}\right)-a_{h}\left(R_{h} u, I_{h}^{*} v_{h}\right)\right)+a_{h}\left(u, I_{h}^{*} v_{h}\right)-\bar{a}_{h}\left(u_{h}, I_{h}^{*} v_{h}\right) \\
& =\left(\bar{a}_{h}\left(R_{h} u, I_{h}^{*} v_{h}\right)-a_{h}\left(R_{h} u, I_{h}^{*} v_{h}\right)\right)-\left(u_{t}-u_{H, t}, I_{h}^{*} v_{h}\right)+\left(f(u)-f\left(u_{H}\right), I_{h}^{*} v_{h}\right) \\
& =S_{1}-S_{2}+S_{3} .
\end{aligned}
$$

By Lemmas 4.1, 4.2, and (2.8), we have

$$
\begin{aligned}
\left|S_{1}\right| & =\left|\bar{a}_{h}\left(R_{h} u, I_{h}^{*} v_{h}\right)-a_{h}\left(R_{h} u, I_{h}^{*} v_{h}\right)\right| \\
& =\left|a\left(R_{h} u, v_{h}\right)-a_{h}\left(R_{h} u, I_{h}^{*} v_{h}\right)\right| \\
& \leq C h^{2}\left(h^{-1}\left\|u-R_{h} u\right\|_{1}+\|u\|_{2}\right)\left\|v_{h}\right\|_{1} \\
& \leq C h^{2}\|u\|_{2}\left\|v_{h}\right\|_{1} .
\end{aligned}
$$

To estimate $S_{2}$, we rewrite $S_{2}$ as follows

$$
\begin{aligned}
S_{2} & =\left(u_{t}-u_{H, t}, I_{h}^{*} v_{h}\right) \\
& =\left(u_{t}-u_{H, t}, I_{h}^{*}\left(v_{h}-I_{H} v_{h}\right)\right)+\left(u_{t}-u_{H, t}, I_{H}^{*}\left(I_{H} v_{h}\right)\right)+\left(u_{t}-u_{H, t}, I_{h}^{*}\left(I_{H} v_{h}\right)-I_{H}^{*}\left(I_{H} v_{h}\right)\right) \\
& =S_{21}+S_{22}+S_{23},
\end{aligned}
$$

where $I_{H}: C(\Omega) \rightarrow S_{H}$ is the general linear interpolation operator satisfying (see, e.g., [3,12])

$$
\left\|v-I_{H} v\right\|_{m, p} \leq C H^{s-m}\|v\|_{s, p}
$$

for $0 \leq m \leq s \leq 2$ and $v \in W_{p}^{s}(\Omega), 1 \leq p \leq \infty$. It is easy to see that $\left\|I_{H} v\right\|_{1} \leq C\|v\|_{1}$. From (2.3), Lemma 4.3, and (4.15), we have

$$
\begin{aligned}
\left|S_{21}\right| & =\left|\left(u_{t}-u_{H, t}, I_{h}^{*}\left(v_{h}-I_{H} v_{h}\right)\right)\right| \\
& \leq\left\|u_{t}-u_{H, t}\right\|_{0}\left\|I_{h}^{*}\left(v_{h}-I_{H} v_{h}\right)\right\|_{0} \\
& \leq C H\left\|v_{h}-I_{H} v_{h}\right\|_{0} \\
& \leq C H^{2}\left\|v_{h}\right\|_{1} .
\end{aligned}
$$

Using Lemma 4.5, we obtain

$$
\begin{aligned}
\left|S_{22}\right| & =\left|\left(u_{t}-u_{H, t}, I_{H}^{*}\left(I_{H} v_{h}\right)\right)\right| \\
& \leq C H^{2}\left\|I_{H} v_{h}\right\|_{1} \leq C H^{2}\left\|v_{h}\right\|_{1} .
\end{aligned}
$$

It follows from Lemma 4.3, a triangle inequality and (2.2) that

$$
\begin{aligned}
\left|S_{23}\right| & =\left|\left(u_{t}-u_{H, t}, I_{h}^{*}\left(I_{H} v_{h}\right)-I_{H}^{*}\left(I_{H} v_{h}\right)\right)\right| \\
& \leq\left\|u_{t}-u_{H, t}\right\|_{0}\left\|I_{h}^{*}\left(I_{H} v_{h}\right)-I_{H}^{*}\left(I_{H} v_{h}\right)\right\|_{0} \\
& \leq C H\left(\left\|I_{h}^{*}\left(I_{H} v_{h}\right)-I_{H} v_{h}\right\|_{0}+\left\|I_{H}^{*}\left(I_{H} v_{h}\right)-I_{H} v_{h}\right\|_{0}\right) \\
& \leq C H\left(h\left\|I_{H} v_{h}\right\|_{1}+H\left\|I_{H} v_{h}\right\|_{1}\right) \\
& \leq C H(h+H)\left\|v_{h}\right\|_{1} .
\end{aligned}
$$


From the above inequalities, we obtain an estimate of $S_{2}$ :

$$
\left|S_{2}\right| \leq C H(h+H)\left\|v_{h}\right\|_{1} \leq C H^{2}\left\|v_{h}\right\|_{1} .
$$

Similarly, we have

$$
\begin{aligned}
\left|S_{3}\right|= & \left|\left(f(u)-f\left(u_{H}\right), I_{h}^{*} v_{h}\right)\right| \\
\leq & \left|\left(f(u)-f\left(u_{H}\right), I_{h}^{*}\left(v_{h}-I_{H} v_{h}\right)\right)\right|+\left|\left(f(u)-f\left(u_{H}\right), I_{H}^{*}\left(I_{H} v_{h}\right)\right)\right| \\
& +\left|\left(f(u)-f\left(u_{H}\right), I_{h}^{*}\left(I_{H} v_{h}\right)-I_{H}^{*}\left(I_{H} v_{h}\right)\right)\right| \\
\leq & C H\left\|f(u)-f\left(u_{H}\right)\right\|_{0}\left\|v_{h}\right\|_{1}+C H^{2}\left\|I_{H} v_{h}\right\|_{1} \\
& +C(h+H)\left\|f(u)-f\left(u_{H}\right)\right\|_{0}\left\|v_{h}\right\|_{1} \\
\leq & C H\left\|u-u_{H}\right\|_{1}\left\|v_{h}\right\|_{1}+C H^{2}\left\|v_{h}\right\|_{1}+C(h+H)\left\|u-u_{H}\right\|_{1}\left\|v_{h}\right\|_{1} \\
\leq & C H^{2}\left\|v_{h}\right\|_{1},
\end{aligned}
$$

where we have used (4.9), Lemma 4.4, and Theorem 3.6. Combining (4.14), (4.16) and (4.17), we have

$$
\left|\bar{a}_{h}\left(R_{h} u-u_{h}, I_{h}^{*} v_{h}\right)\right| \leq C H^{2}\left\|v_{h}\right\|_{1} .
$$

Taking $v_{h}=R_{h} u-u_{h}$ and using Lemma 3.1 (coercivity), we obtain

$$
\left\|R_{h} u-u_{h}\right\|_{1} \leq C H^{2} .
$$

It follows from (2.8) and a triangle inequality that

$$
\left\|u-u_{h}\right\|_{1} \leq\left\|R_{h} u-u_{h}\right\|_{1}+\left\|u-R_{h} u\right\|_{1} \leq C\left(H^{2}+h\right),
$$

which yields the desired result.

Remark 4.7. From Theorem 4.6, we see that if $h=\mathcal{O}\left(H^{2}\right)$, then the highest possible convergence rate in the $H^{1}$ norm for the postprocessing FVE method is $\mathcal{O}\left(H^{2}\right)$. Thus the postprocessing procedure improves the convergence rate over the standard FVE method error estimate in the $H^{1}$ norm, which is only $\mathcal{O}(H)$ (see Thm. 3.6), by one order. Since the mesh refinement is performed only at the final time $T$, the method increases the accuracy of the standard FVE approximation at low extra computational costs.

Remark 4.8. We consider the spatial discretization to focus on postprocessing. In practical computations, the method should be combined with a time-stepping algorithm. Let $N$ be a positive integer. Consider a temporal discretization $0=t_{0}<t_{1}<\ldots<t_{N}=T$ and set $u^{n}=u\left(\cdot, t_{n}\right)(0 \leq n \leq N)$. Then an implicit backward Euler postprocessing procedure is given by

(1) Find $u_{H}^{n} \in S_{H}$ such that for any $v_{H} \in S_{H}$,

$$
\left(\frac{u_{H}^{n}-u_{H}^{n-1}}{t_{n}-t_{n-1}}, I_{H}^{*} v_{H}\right)+a_{H}\left(u_{H}^{n}, I_{H}^{*} v_{H}\right)=\left(f^{n}\left(\mathbf{x}, u_{H}^{n}\right), I_{H}^{*} v_{H}\right), \quad 1 \leq n \leq N
$$

with $u_{H}^{0}=R_{H} u_{0}$. 
TABLE 1. $H^{1}$ and $L^{2}$ errors and convergence rates on the coarser grid.

\begin{tabular}{lllll}
\hline$N$ & $H^{1}$-norm error & Rate & $L^{2}$-norm error & Rate \\
\hline 2 & $1.1909 \times 10^{-1}$ & - & $6.7219 \times 10^{-2}$ & - \\
4 & $9.0843 \times 10^{-2}$ & 0.40 & $1.5746 \times 10^{-2}$ & 2.09 \\
8 & $5.6665 \times 10^{-2}$ & 0.68 & $3.8616 \times 10^{-3}$ & 2.03 \\
16 & $3.1461 \times 10^{-2}$ & 0.85 & $9.5043 \times 10^{-4}$ & 2.02 \\
32 & $1.6556 \times 10^{-2}$ & 0.93 & $2.7318 \times 10^{-4}$ & 1.80 \\
64 & $8.5047 \times 10^{-3}$ & 0.96 & $1.0567 \times 10^{-2}$ & 1.37 \\
\hline
\end{tabular}

TABle 2. Postprocessed $H^{1}$ errors and convergence rates on the finer grids.

\begin{tabular}{lllll}
\hline$N$ & $h=1 / N^{2}$ & Rate & $h=1 /(3 N)$ & Rate \\
\hline 2 & $9.0458 \times 10^{-2}$ & - & $7.0077 \times 10^{-2}$ & - \\
4 & $3.1494 \times 10^{-2}$ & 1.52 & $4.0542 \times 10^{-2}$ & 0.79 \\
8 & $8.5010 \times 10^{-3}$ & 1.89 & $2.1721 \times 10^{-2}$ & 0.90 \\
16 & $2.2445 \times 10^{-3}$ & 1.92 & $1.1236 \times 10^{-2}$ & 0.95 \\
32 & $5.7212 \times 10^{-4}$ & 1.97 & $5.7134 \times 10^{-3}$ & 0.98 \\
\hline
\end{tabular}

(2) Find $u_{h} \in S_{h}$ such that for any $v_{h} \in S_{h}$,

$$
\bar{a}_{h}\left(u_{h}, I_{h}^{*} v_{h}\right)=-\left(\frac{u_{H}^{N}-u_{H}^{N-1}}{t_{N}-t_{N-1}}, I_{h}^{*} v_{h}\right)+\left(f^{N}\left(\mathbf{x}, u_{H}^{N}\right), I_{h}^{*} v_{h}\right) .
$$

Of course, higher order temporal discretization methods such as the Runge-Kutta methods or multistep methods can also be used. On the other hand, from a practical point of view, we just need to choose $h<H$ to obtain a considerable error reduction in spite of the demanding requirement $h=\mathcal{O}\left(H^{2}\right)$.

\section{NumericAl EXPERIMENTS}

In this section, we present numerical experiments to illustrate the theoretical results presented in the previous sections. In particular, our main interest is to verify Theorems 3.6 and 4.6. We consider the following parabolic equation with a quadratic nonlinearity,

$$
u_{t}-\nabla \cdot\left(\frac{1}{1+|\mathbf{x}|^{2}} \nabla u\right)+u^{2}=f(\mathbf{x}, t), \quad \text { in } \Omega \times(0, T]
$$

with a homogeneous Dirichlet boundary condition. The domain is $\Omega=[0,1]^{2}$, the final time is $T=1$, the exact solution is $u(x, y, t)=\mathrm{e}^{-t / 2} \sin (\pi x) \sin (\pi y)$, and the right hand side $f(\mathbf{x}, t)$ is computed accordingly.

The domain $\Omega$ is partitioned into $N$ uniform pieces in each direction and then each rectangle is divided into two triangles, resulting in a mesh with size $H=1 / N$. The finite element space $S_{H}$ is built on the coarser grid $\mathcal{T}_{H}$ with $N=2,4,8,16,32,64$. We use the backward Euler temporal formula with a relatively small time step $\Delta t=10^{-3}$, so that the dominant error will be the spatial error. Table 1 lists the errors in the $H^{1}$ and $L^{2}$ norms and also the convergence rates at $t=T$, respectively. The results are in accordance with the estimates obtained in Theorem 3.6 for $N \leq 32$. It is not a surprise to see that the $L^{2}$ error convergence rate for $N=64$ drops significantly. This is due to the fact that the expected $L^{2}$ error for the backward Euler 
fully discrete scheme is of order $O\left(\Delta t+H^{2}\right)$ and the error is dominated by the temporal approximation as $N$ increases.

To illustrate the theoretical findings in Theorem 4.6, we compute the postprocessing FVE approximation at $T$ on two finer grids with $h=H^{2}$ and $h=H / 3$, respectively. Table 2 shows that, if $h=H^{2}$, then the $H^{1}$ convergence rate is close to second order; but if $h=H / 3$, the $H^{1}$ convergence rate is nearly unchanged, although the error is smaller when compared with the results in Table 1. We also find that the $H^{1}$ errors in Table 1 for $N=4,16,64$ are approximately the same as those in Table 2 for $N=2,4,8$, when $h=H^{2}$ is used. This means that one step postprocessing on a finer grid can yield the same accuracy as a standard all-time-level FVE computation on the same grid. Therefore, these results confirm Theorem 4.6 and Remark 4.7.

Acknowledgements. The authors would like to express their sincere thanks to the referees for their careful reading and valuable comments and suggestions.

\section{REFERENCES}

[1] R. Adams and J.J.F. Fournier, Sobolev Spaces. Academic Press, New York (2003).

[2] C. Bi and V. Ginting, Two-grid finite volume element method for linear and nonlinear elliptic problems. Numer. Math. 107 (2007) $177-198$.

[3] S.C. Brenner and L.R. Scott, The Mathematical Theory of Finite Element Methods. Springer-Verlag, New York, 2nd edn., (2002).

[4] Z. Cai, On the finite volume element method. Numer. Math. 58 (1991) 713-735.

[5] Z. Cai, J. Mandel and S. McCormick, The finite volume element method for diffusion equations on general triangulations. SIAM J. Numer. Anal. 28 (1991) 392-402.

[6] C. Carstensen, R. Lazarov and S. Tomov, Explicit and averaging a posteriori error estimates for adaptive finite volume methods. SIAM J. Numer. Anal. 42 (2005) 2496-2521.

[7] P. Chatzipantelidis and R.D. Lazarov, Error estimates for a finite volume element method for elliptic PDEs in nonconvex polygonal domains. SIAM J. Numer. Anal. 42 (2004) 1932-1958.

[8] P. Chatzipantelidis, R.D. Lazarov and V. Thomée, Error estimates for a finite volume element method for parabolic equations in convex polygonal domains. Numer. Meth. PDEs 20 (2004) 650-674.

[9] S.H. Chou and D.Y. Kwak, Multigrid algorithms for a vertex-centered covolume method for elliptic problems. Numer. Math. 90 (2002) 459-486.

[10] S.H. Chou and Q. Li, Error estimates in $L^{2}, H^{1}$ and $L^{\infty}$ in covolume methods for elliptic and parabolic problems: a unified approach. Math. Comp. 69 (2000) 103-120.

[11] S.H. Chou, D.Y. Kwak and Q. Li, $L^{p}$ error estimates and superconvergence for covolume or finite volume element methods. Numer. Meth. PDEs 19 (2003) 463-486.

[12] P.G. Ciarlet, The Finite Element Method for Elliptic Problems. North-Holland, Amsterdam (1978).

[13] C.N. Dawson, M.F. Wheeler and C.S. Woodward, A two-grid finite difference scheme for nonlinear parabolic equations. SIAM J. Numer. Anal. 35 (1998) 435-452.

[14] J. de Frutos and J. Novo, Postprocessing the linear finite element method. SIAM J. Numer. Anal. 40 (2002) 805-819.

[15] R.E. Ewing, T. Lin and Y. Lin, On the accuracy of the finite volume element method based on piecewise linear polynomials. SIAM J. Numer. Anal. 39 (2002) 1865-1888.

[16] R. Eymard, T. Gallouët and R. Herbin, Finite Volume Methods: Handbook of Numerical Analysis. North-Holland, Amsterdam (2000).

[17] M. Feistauer, J. Felcman, M. Lukáčová-Medvidová and G. Warnecke, Error estimates of a combined finite volume-finite element method for nonlinear convection-diffusion problems. SIAM J. Numer. Anal. 36 (1999) 1528-1548.

[18] B. García-Archilla, J. Novo and E.S. Titi, Postprocessing the Galerkin method: a novel approach to approximate inertial manifolds. SIAM J. Numer. Anal. 35 (1998) 941-972.

[19] B. García-Archilla and E.S. Titi, Postprocessing the Galerkin method: the finite element case. SIAM J. Numer. Anal. 37 (2000) 470-499.

[20] D. Henry, Geometric theory of semilinear parabolic equations, Lecture Notes in Mathematics 840. Springer-Verlag, New York (1989).

[21] A. Lasis and E. Süli, $h p$-version discontinuous Galerkin finite element method for semilinear parabolic problems. SIAM J. Numer. Anal. 45 (2007) 1544-1569.

[22] R. Li, Z. Chen and W. Wu, Generalized Difference Methods for Differential Equations: Numerical Analysis of Finite Volume Methods. Marcel Dekker, New York (2000). 
[23] X. Ma, S. Shu and A. Zhou, Symmetric finite volume discretizations for parabolic problems. Comput. Methods Appl. Mech. Engrg. 192 (2003) 4467-4485.

[24] M. Marion and J.C. Xu, Error estimates on a new nonlinear Galerkin method based on two-grid finite elements. SIAM J. Numer. Anal. 32 (1995) 1170-1184.

[25] H. Rui, Symmetric modified finite volume element methods for self-adjoint elliptic and parabolic problems. J. Comput. Appl. Math. 146 (2002) 373-386.

[26] A.H. Schatz, V. Thomée and L. Wahlbin, Maximum norm stability and error estimates in parabolic finite element equations. Comm. Pure Appl. Math. 33 (1980) 265-304.

[27] R.K. Sinha and J. Geiser, Error estimates for finite volume element methods for convection-diffusion-reaction equations. Appl. Numer. Math. 57 (2007) 59-72.

[28] R. Temam, Infinite Dimensional Dynamical Systems in Mechanics and Physics, Applied Mathematical Sciences 68. SpringerVerlag, Berlin (1988).

[29] V. Thomée, Galerkin Finite Element Methods for Parabolic Problems. Springer-Verlag, Berlin (1997).

[30] V. Thomée and L. Wahlbin, On Galerkin methods in semilinear parabolic problems. SIAM J. Numer. Anal. 12 (1975) $378-389$.

[31] Y. Yan, Postprocessing the finite element method for semilinear parabolic problems. SIAM J. Numer. Anal. 44 (2006) 16811702.

[32] M. Yang, A second-order finite volume element method on quadrilateral meshes for elliptic equations. ESAIM: M2AN 40 (2006) 1053-1067.

[33] X. Ye, A discontinuous finite volume method for the Stokes problems. SIAM J. Numer. Anal. 44 (2006) $183-198$.

[34] S. Zhang, On domain decomposition algorithms for covolume methods for elliptic problems. Comput. Methods Appl. Mech. Engrg. 196 (2006) 24-32. 